\title{
Tensor fields defined by Lax representations
}

Alexander V. Balandin

To cite this article: Alexander V. Balandin (2016) Tensor fields defined by Lax representations, Journal of Nonlinear Mathematical Physics 23:3, 323-334, DOI: https://doi.org/10.1080/14029251.2016.1199494

To link to this article: https://doi.org/10.1080/14029251.2016.1199494

Published online: 04 January 2021 


\title{
Tensor fields defined by Lax representations *
}

\author{
Alexander V. Balandin ${ }^{\dagger}$ \\ Department of Algebra, Geometry and Discrete Mathematics, Lobachevsky State University, 23 Prospekt Gagarina, \\ Nizhni Novgorod 603950, Russia \\ balandin31@ rambler.ru *
}

Received 7 January 2016

Accepted 15 March 2016

\begin{abstract}
In this paper, some properties of tensor fields constructed by the Lax representation of chiral-type systems are investigated.

Keywords: chiral-type systems; Lax representation; characteristics of conservation laws.

2010 Mathematics Subject Classification: 35Q51,37K10,37K05
\end{abstract}

\section{Introduction}

Chiral-type systems (see, for example, [4]) are the systems of partial differential equations of the form

$$
\Delta^{\alpha} \equiv U_{x y}^{\alpha}+G_{\beta \gamma}^{\alpha} U_{x}^{\beta} U_{y}^{\gamma}+Q^{\alpha}=0 .
$$

Here and further, the Greek indices range from 1 to $n$ and the subscripts denote partial derivatives with respect to the independent variables $x$ and $y$. The coefficients $G_{\beta \gamma}^{\alpha}, Q^{\alpha}$ are assumed to be smooth functions of the variables $U^{1}, U^{2}, \ldots, U^{n}$. The summation rule over the repeated indices is also assumed.

Further on, the covariant derivatives w.r.t. the connection defined by the coefficients $G_{\beta \gamma}^{\alpha}$ are denoted by $\nabla_{\delta}$.

The Euler-Lagrange equations of the form (1.1) are called a nonlinear generalized sigma model.

Following [7], recall that the characteristic of the conservation law $L=\left(L_{1}, L_{2}\right)$ of the system (1.1) is a set of functions $R=\left\{R_{\alpha}\right\}$ such that

$$
\operatorname{Div} L=D_{x} L_{1}+D_{y} L_{2}=R_{\alpha} \Delta^{\alpha},
$$

where $D_{x}$ and $D_{y}$ are the total derivatives w.r.t. $x, y$, and $\Delta^{\alpha}$ denotes the 1.h.s. of Eq. (1.1).

We understand integrable systems as the systems admitting a Lax representation also called zero-curvature representations.

\footnotetext{
${ }^{*}$ Tensor fields defined by Lax representations

†Department of Algebra, Geometry and Discrete Mathematics, Lobachevsky State University, 23 Prospekt Gagarina, Nizhny Novgorod, 603950, Russia

†balandin31@ rambler.ru
} 
In the sequel, we assume that the system (1.1) admits the matrix $\mathfrak{g}$-valued Lax representation of the form:

$$
D_{y} \widetilde{A}-D_{x} \widetilde{B}+[\widetilde{A}, \widetilde{B}]=S_{\alpha} \Delta^{\alpha},
$$

where

$$
\begin{gathered}
\widetilde{A}=A_{\alpha} U_{x}^{\alpha}+\lambda M, \quad \widetilde{B}=B_{\alpha} U_{y}^{\alpha}+\frac{1}{\lambda} N, \\
S_{\alpha}=A_{\alpha}-B_{\alpha},
\end{gathered}
$$

$\lambda$ is an irreducible parameter, $A, B, M, N$ are smooth functions of the variables $U^{1}, U^{2}, \ldots, U^{n}$, taking values in a matrix Lie algebra $\mathfrak{g}$.

It is easy to see, collecting the terms by $\lambda, \frac{1}{\lambda}$, that $M, N, A_{\alpha}, B_{\alpha}$ satisfy the following conditions

$$
\begin{gathered}
M_{, \alpha}=\left[B_{\alpha}, M\right], \\
N_{, \alpha}=\left[A_{\alpha}, N\right] . \\
Q^{\alpha} S_{\alpha}=[M, N], \\
A_{\alpha, \beta}-B_{\beta, \alpha}+\left[A_{\alpha}, B_{\beta}\right]-S_{\gamma} G_{\alpha \beta}^{\gamma}=0 .
\end{gathered}
$$

Here and further, comma denotes the partial derivatives, that is, $P_{, \alpha}=\frac{\partial P}{\partial U^{\alpha}}$.

The set of functions $S_{\alpha}$ in Eq. (1.2) is referred to as a characteristic element of the Lax representation [6]. In the sequel, it is assumed that $S_{1}, S_{2}, \ldots, S_{n}$ are linear independent.

Remark 1.1. Note that the functions $M, N$, and the characteristic element $S_{\alpha}$ of the Lax representation (1.2), (1.3) are transformed under a gauge transformation by the formulas

$$
M \rightarrow T^{-1} M T, N \rightarrow T^{-1} N T, S_{\alpha} \rightarrow T^{-1} S_{\alpha} T .
$$

Thus, the functions of the form $f(S_{\alpha_{1}}, S_{\alpha_{2}}, \ldots S_{\alpha_{p}}, \underbrace{M, M, \ldots, M}_{r}, \underbrace{N, N, \ldots, N}_{s})$ are well defined tensor fields for an arbitrary ad-invariant symmetric $(p+r+s)$-form on $\mathfrak{g}$. It turns out that these tensor fields carry important information about the system under consideration.

It was mentioned in [1] that for an arbitrary ad-invariant symmetric $(k+p)$-form on $\mathfrak{g}$, tensor fields of the form

$$
F_{\alpha_{1} \alpha_{2} \ldots \alpha_{k}}=f(S_{\alpha_{1}}, S_{\alpha_{2}}, \ldots S_{\alpha_{k}}, \underbrace{M, M, \ldots, M}_{p}),(k>0, p \geq 0)
$$

are Killing fields, that is

$$
\nabla_{(\beta} F_{\left.\alpha_{1} \alpha_{2} \ldots \alpha_{k}\right)}=0 .
$$

Here the proof of it is given (Proposition 2.1). 
The important case $k=1$ of such tensors was considered in [2]. It turns out that the sets $F_{\alpha}$ defined by the expressions

$$
F_{\alpha}=f\left(S_{\alpha}, M, M, \ldots M\right)
$$

are characteristics of the zero order of conservation laws of system (1.1).

In this paper, in a similar way, we investigate the meaning of the tensors

$$
K_{\alpha_{1} \alpha_{2} \ldots \alpha_{k}}=f\left(\left[S_{\alpha_{1}}, M\right],\left[S_{\alpha_{2}}, M\right], \ldots,\left[S_{\alpha_{k}}, M\right], M, \ldots, M\right) .
$$

It is shown that covariant derivatives of these tensors vanish (Theorem 2.1). According to well known result, these tensors for $k=2$ define conservation laws $K_{\alpha \beta} U_{x}^{\alpha} U_{x}^{\beta} d x$ for the system

$$
\Delta^{\alpha} \equiv U_{x y}^{\alpha}+G_{\beta \gamma}^{\alpha} U_{x}^{\beta} U_{y}^{\gamma}=0
$$

that is, the sets $K_{\alpha \beta} U_{x}^{\alpha}$ form the characteristics of the first order of conservation laws for system (1.8).

Note that the last result is not valid for the general case of system (1.1) with non vanishing $Q^{\alpha}$ (Example 2.1).

However, the sets $K_{\alpha \beta} U_{x}^{\alpha}$ form the characteristics of the first order of conservation laws for 3 -dimensional Lie algebras $\mathfrak{g}$. This result was announced in [2]. Here the full proof of it is given (Theorem 2.2).

Remark 1.2. It is obvious that the results obtained from the above mentioned ones are valid also if we change $x \leftrightarrow y, M \leftrightarrow N, G_{\beta \gamma}^{\alpha} \leftrightarrow G_{\beta \gamma}^{\alpha}-2 G_{[\beta \gamma]}^{\alpha}$.

\section{Main theorems}

Proposition 2.1. Let the system (1.1) admit the matrix $\mathfrak{g}$-valued Lax representation of the form (1.3) and $f$ be a symmetric ad-invariant p-form $f$ on Lie algebra $\mathfrak{g}$. Then the tensor field

$$
F_{\alpha_{1} \alpha_{2} \ldots \alpha_{k}}=f(S_{\alpha_{1}}, S_{\alpha_{2}}, \ldots S_{\alpha_{k}}, \underbrace{M, M, \ldots, M}_{p}),(k>0, p \geq 0)
$$

satisfy the condition

$$
\nabla_{(\beta} F_{\left.\alpha_{1} \alpha_{2} \ldots \alpha_{k}\right)}=0
$$

that is, $F_{\alpha_{1} \alpha_{2} \ldots \alpha_{k}}$ is a Killing field.

Proof. Rewrite Eq. (1.7) in the form

$$
\nabla_{\beta} S_{\alpha}=S_{\alpha, \beta}-S_{\gamma} G_{\alpha \beta}^{\gamma}=\left[B_{\beta}, S_{\alpha}\right]+D_{\alpha \beta},
$$

where

$$
D_{\alpha \beta}=\left[B_{\beta}, B_{\alpha}\right]+2 B_{[\beta, \alpha]} .
$$

Then, one can obtain 


$$
\begin{gathered}
\nabla_{\beta} F_{\alpha_{1} \alpha_{2} \ldots \alpha_{k}}=f(\nabla_{\beta} S_{\alpha_{1}}, S_{\alpha_{2}}, \ldots S_{\alpha_{k}}, \underbrace{M, M, \ldots, M}_{p}) \\
+f(S_{\alpha_{1}}, \nabla_{\beta} S_{\alpha_{2}}, \ldots S_{\alpha_{k}}, \underbrace{M, M, \ldots, M}_{p}) \\
+\ldots+f(S_{\alpha_{1}}, S_{\alpha_{2}}, \ldots, \nabla_{\beta} S_{\alpha_{k}}, \underbrace{M, M, \ldots, M}_{p})+p f(S_{\alpha_{1}}, S_{\alpha_{2}}, \ldots S_{\alpha_{k}}, \underbrace{M_{, \beta}, M, \ldots, M}_{p}) .
\end{gathered}
$$

Using Ad-invariancy of form $f$ and Eq. (1.4),(2.1), one can see that

$$
\begin{gathered}
\nabla_{\beta} F_{\alpha_{1} \alpha_{2} \ldots \alpha_{k}}=f(D_{\alpha_{1} \beta}, S_{\alpha_{2}}, \ldots S_{\alpha_{k}}, \underbrace{M, M, \ldots, M}_{p})+f(S_{\alpha_{1}}, D_{\alpha_{2} \beta}, \ldots S_{\alpha_{k}}, \underbrace{M, M, \ldots, M}_{p}) \\
+f(S_{\alpha_{1}}, S_{\alpha_{2}}, \ldots D_{\alpha_{k} \beta}, \underbrace{M, M, \ldots, M}_{p}) .
\end{gathered}
$$

Symmetrizing Eq. (2.3) and taking into account that $D_{(\alpha \beta)}=0$, we finish the proof.

Denote the $\alpha$-th Euler operator by

$$
E_{\alpha}=\sum_{J}(-D)_{J}\left(\frac{\partial}{\partial U_{J}^{\alpha}}\right)
$$

where the sum extending over all multi-indices $J=\left(j_{1}, j_{2}\right)$.

To proceed we need the following lemma.

Lemma 2.1. Let $R$ be a function of the form:

$$
R=K_{\alpha \beta} U_{x y}^{\alpha} U_{x}^{\beta}+L_{\alpha \beta \gamma} U_{x}^{\alpha} U_{x}^{\beta} U_{y}^{\gamma}+W_{\alpha} U_{x}^{\alpha},
$$

where $K_{\alpha \beta}, L_{\alpha \beta \gamma}, W_{\alpha}$ are functions of $U^{1}, U^{2}, \ldots, U^{n}$.

Then, the equations

$$
E_{\mu}(R)=0
$$

are equivalent to the following conditions:

$$
\begin{gathered}
K_{[\alpha \mu]}=0, \\
K_{\mu \alpha, \beta}-2 L_{(\mu \alpha) \beta}=0, \\
W_{[\alpha, \mu]}=0 .
\end{gathered}
$$

Here and further, the comma denotes the partial derivatives, that is, $P_{, \alpha}=\frac{\partial P}{\partial U^{\alpha}}$. 
Proof. The proof is obtained by direct computation. Collecting the terms by $U_{x x y}^{\alpha}, U_{x y}^{\alpha} U_{y}^{\beta}$, $U_{x}^{\alpha} U_{x}^{\beta} U_{y}^{\gamma}, U_{x}^{\alpha}$ in the expression $E_{\mu}(R)$ and taking into account the condition $E_{\mu}(R)=0$, we obtain Eq. (2.4),(2.5), the equation $K_{\alpha \beta,[\mu, \gamma]}=0$ (which is an identity), and Eq. (2.6).

Remark 2.1. Assuming that

$$
L_{\alpha \beta \gamma}=K_{\alpha \mu} G_{\beta \gamma}^{\mu}, W_{\alpha}=K_{\alpha \beta} Q^{\beta} .
$$

Then one can easily verify that Eq. (2.6) can be rewritten in the form

$$
\left(Q^{\alpha} K_{\alpha \beta}\right)_{, \gamma}-\left(Q^{\alpha} K_{\alpha \gamma}\right)_{, \beta}=0,
$$

and Eq. (2.4),(2.5) are equivalent to the condition

$$
\nabla_{\gamma} K_{\alpha \beta}=0
$$

Theorem 2.1. Let the system (1.1) admit the matrix $\mathfrak{g}$-valued Lax representation of the form (1.3) and $f$ be a symmetric ad-invariant p-form $f$ on Lie algebra $\mathfrak{g}$.

Then the tensor field

$$
K_{\alpha_{1} \alpha_{2} \ldots \alpha_{k}}=f(\left[S_{\alpha_{1}}, M\right],\left[S_{\alpha_{2}}, M\right], \ldots,\left[S_{\alpha_{k}}, M\right], \underbrace{M, \ldots, M}_{p-k})
$$

satisfies the condition

$$
\nabla_{\gamma} K_{\alpha_{1} \alpha_{2} \ldots \alpha_{k}}=0
$$

We will give the proof of the theorem in Appendix.

Corollary 2.1. Let the system (1.8) admit the matrix $\mathfrak{g}$-valued Lax representation of the form (1.3). Then for every symmetric ad-invariant $p$-form $f$ on Lie algebra $\mathfrak{g}$ the set

$$
Y_{\alpha}=f(\left[S_{\alpha}, M\right],\left[S_{\beta}, M\right], \underbrace{M, M, \ldots, M}_{p-2}) U_{x}^{\beta}
$$

is the characteristic of the conservation law $\theta=\frac{1}{2} f(\left[S_{\alpha}, M\right],\left[S_{\beta}, M\right], \underbrace{M, M, \ldots, M}_{p-2}) U_{x}^{\alpha} U_{x}^{\beta} d x$.

Proof. The proof follows from the well known result that $K_{\alpha \beta} U_{x}^{\alpha} U_{x}^{\beta} d x$ is an integral of system (1.8) iff $\nabla_{\gamma} K_{\alpha \beta}=0$.

Corollary 2.2. Let n-component system (1.1) admit $\mathfrak{g}$-valued Lax representation of the form (1.3), where $\mathfrak{g}$ is compact algebra Lie of rank $l$ and $l<n$. Assume that $M$ is a regular element of Lie algebra $\mathfrak{g}$ in a point $P_{0}$.

Then the covariant constant tensor field $K_{\alpha \beta}$ defined by

$$
K_{\alpha \beta}=f\left(\left[S_{\alpha}, M\right],\left[S_{\beta}, M\right]\right),
$$

where $f$ is a Killing form on $\mathfrak{g}$, does not vanish at point $P_{0}$. 
Proof. One can obtain the proof taking into account the linear independence of $S_{\alpha}$, the compactness of $\mathfrak{g}$, and reasons of the dimensionality.

Remark 2.2. It is well known that the Euler-Lagrange system for Lagrangian

$$
L=g_{\alpha \beta}\left(U^{\gamma}\right) U_{x}^{\alpha} U_{y}^{\beta}+Q\left(U^{\gamma}\right)
$$

where $g_{[\alpha \beta]}=0, \operatorname{det}\left\|g_{\alpha \beta}\right\| \neq 0$, admits the integrals $g_{\alpha \beta} U_{x}^{\alpha} U_{x}^{\beta} d x, g_{\alpha \beta} U_{y}^{\alpha} U_{y}^{\beta} d y$. Thus, if tensor field $f(\left[S_{\alpha}, M\right],\left[S_{\beta}, M\right], \underbrace{M, M, \ldots, M}_{p-2})$ is up to a constant proportional to the metric $g_{\alpha \beta}$ determined by Lagrangian $L$, then the set $f(\left[S_{\alpha}, M\right],\left[S_{\beta}, M\right], \underbrace{M, M, \ldots, M}_{p-2}) U_{x}^{\alpha}$ is the characteristic of conservation law. Note that no examples of such systems admitting the Lax representation with $\operatorname{dim} \mathfrak{g}>3$ are known to the author.

Remark 2.3. Corollary 2.1 could not be generalized to the case of the chiral-type systems (1.1) with non vanishing $Q^{\alpha}$. This illustrates the following example.

Example 2.1. Consider the 3-component variational system

$$
\begin{gathered}
\Delta^{1}=U_{x y}^{1}-a U^{2} e^{2 U^{1}}+b U^{3} e^{-2 U^{1}}=0, \\
\Delta^{2}=U_{x y}^{2}-b \psi^{-1} e^{-2 U^{1}}-\psi U^{3} U_{x}^{2} U_{y}^{2}=0, \\
\Delta^{3}=U_{x y}^{3}-a \psi^{-1} e^{2 U^{1}}-\psi U^{2} U_{x}^{3} U_{y}^{3}=0,
\end{gathered}
$$

where $\psi=\left(U^{2} U^{3}+c\right)^{-1}$ and $a, b, c$ are arbitrary constants, and $a^{2}+b^{2} \neq 0$.

This system is the Euler system for Lagrangian

$$
L=2 U_{x}^{1} U_{y}^{1}+\psi\left(U_{x}^{2} U_{y}^{3}+U_{x}^{3} U_{y}^{2}\right)+2 a U^{2} e^{2 U^{1}}+2 b U^{3} e^{-2 U^{1}}
$$

and admits the Lax representation which takes values in $s l(3)$, where [4]:

$$
\begin{gathered}
\widetilde{A}=\left(\begin{array}{ccc}
-\frac{U_{x}^{2} U^{3}}{3\left(U^{2} U^{3}+c\right)} & -b c^{-1} U^{3} e^{-2 U^{1}} & 0 \\
\lambda a U^{2} e^{2 U^{1}} & -\frac{U_{x}^{2} U^{3}}{3\left(U^{2} U^{3}+c\right)} & \lambda a e^{2 U^{1}} \\
0 & b c^{-1} e^{-2 U^{1}}\left(U^{2} U^{3}+c\right) & \frac{2 U_{x}^{2} U^{3}}{3\left(U^{2} U^{3}+c\right)}
\end{array}\right) \\
\widetilde{B}=\left(\begin{array}{ccc}
-\frac{1}{3}\left(2 U_{y}^{1}+\frac{U^{2} U_{y}^{3}}{U^{2} U^{3}+c}\right) & \lambda-1 & -\frac{U_{y}^{3}}{U^{2} U^{3}+c} \\
-c & \frac{1}{3}\left(4 U_{y}^{1}-\frac{U^{2} U_{y}^{3}}{U^{2} U^{3}+c}\right) & 0 \\
-U_{y}^{2} & 0 & -\frac{1}{3}\left(2 U_{y}^{1}-\frac{2 U^{2} U_{y}^{3}}{U^{2} U^{3}+c}\right)
\end{array}\right) .
\end{gathered}
$$

Co-published by Atlantis Press and Taylor \& Francis 
This Lax representation is not of the form (1.3). In order to construct tensor $K_{\alpha \beta}$ using Eq. (2.8), we rewrite this Lax representation in the gauge equivalent form:

$$
\begin{gathered}
\widetilde{A}=\left(\begin{array}{ccc}
-\frac{U_{x}^{2} U^{3}}{3\left(U^{2} U^{3}+c\right)} & -b c^{-1} U^{3} e^{-2 U^{1}} \lambda & 0 \\
\lambda a U^{2} e^{2 U^{1}} & -\frac{U_{x}^{2} U^{3}}{3\left(U^{2} U^{3}+c\right)} & \lambda a e^{2 U^{1}} \\
0 & b c^{-1} e^{-2 U^{1}\left(U^{2} U^{3}+c\right) \lambda} \frac{2 U_{x}^{2} U^{3}}{3\left(U^{2} U^{3}+c\right)}
\end{array}\right), \\
\widetilde{B}=\left(\begin{array}{ccc}
-\frac{1}{3}\left(2 U_{y}^{1}+\frac{U^{2} U_{y}^{3}}{U^{2} U^{3}+c}\right) & \lambda^{-1} & -\frac{U_{y}^{3}}{U^{2} U^{3}+c} \\
-c \lambda^{-1} & \frac{1}{3}\left(4 U_{y}^{1}-\frac{U^{2} U_{y}^{3}}{U^{2} U^{3}+c}\right) & 0 \\
-U_{y}^{2} & 0 & -\frac{1}{3}\left(2 U_{y}^{1}-\frac{2 U^{2} U_{y}^{3}}{U^{2} U^{3}+c}\right)
\end{array}\right) .
\end{gathered}
$$

Choosing $f(x, y)$ as Killing form $\operatorname{tr}(x y)$, one can find by direct calculation

$$
K=-a b\left(\begin{array}{ccc}
8 & 0 & 0 \\
0 & 0 & \frac{1}{U^{2} U^{3}+c} \\
0 & \frac{1}{U^{2} U^{3}+c} & 0
\end{array}\right) .
$$

Analogously one can obtain by direct calculations:

$$
\widetilde{K}=f\left(\left[S_{\alpha}, N\right],\left[S_{\beta}, N\right]\right)=c\left(\begin{array}{ccc}
4 & 0 & 0 \\
0 & 0 & \frac{1}{U^{2} U^{3}+c} \\
0 & \frac{1}{U^{2} U^{3}+c} & 0
\end{array}\right)
$$

Consider the case $a=b=0$. Then, one can easily see that $\widetilde{K}_{\alpha \beta} U_{y}^{\alpha}$ is the characteristic of the conservation law $c\left[4\left(U_{y}^{1}\right)^{2}+\frac{U_{y}^{2} U_{y}^{3}}{U^{2} U^{3}+c}\right] d y$, in accordance with Corollary 2.1.

In a general case $a \neq 0$ or $b \neq 0$ matrices $K, \widetilde{K}$ are not proportional up to a constant to the metric defined by Lagrangian (2.9), and one can verify that the sets $\widetilde{K}_{\alpha \beta} U_{x}^{\beta}, K_{\alpha \beta} U_{x}^{\beta}$ are not characteristics of the conservation laws.

It is interesting to note that the linear combination $c K+3 a b \widetilde{K}$ is proportional to the metric and defines the characteristic of the conservation law.

Theorem 2.2. Let the system (1.1) admit the Lax representation of the form (1.3) valued in a 3dimensional Lie algebra $\mathfrak{g}$. Then for every symmetric ad-invariant p-form $f$ on Lie algebra $\mathfrak{g}$ the set

$$
Y_{\alpha}=f(\left[S_{\alpha}, M\right],\left[S_{\beta}, M\right], \underbrace{M, M, \ldots, M}_{p-2}) U_{x}^{\beta},
$$

is the characteristic of the conservation law

$$
\theta=\frac{1}{2} f(\left[S_{\alpha}, M\right],\left[S_{\beta}, M\right], \underbrace{M, M, \ldots, M}_{p-2}) U_{x}^{\beta} U_{x}^{\alpha} d x+\frac{(M, M)}{p-1} H d y,
$$

where $H=f(N, \underbrace{M, M, \ldots, M}_{p-1})$ and the round brackets $(M, M)$ denote the scalar product w.r.t. the Killing metric on $\mathfrak{g}$. 
Proof. The proof for the two cases of $s o(3)$ and $s l(2)$ is the same due to the well known isomorphism over $\mathbb{C}$ of these Lie algebras. The only statement we need to prove is the condition (2.7). Denote $Z_{\beta}=Q^{\alpha} K_{\alpha \beta}$. Then, using Eq. (1.6), we have the following equalities

$$
\begin{aligned}
Z_{\beta} & =f(\left[Q^{\alpha} S_{\alpha}, M\right],\left[S_{\beta}, M\right], \underbrace{M, M, \ldots, M}_{p-2})=f([[M, N], M],\left[S_{\beta}, M\right], \underbrace{M, M, \ldots, M}_{p-2}) \\
& =-f(M(M, N),\left[S_{\beta}, M\right], \underbrace{M, M, \ldots, M}_{p-2})+f(N(M, M),\left[S_{\beta}, M\right], \underbrace{M, M, \ldots, M}_{p-2}) .
\end{aligned}
$$

Now, one can see that the first summand vanish due to Ad-invariance of the form $f$. Thus, we obtain

$$
Z_{\beta}=(M, M) f(N,\left[S_{\beta}, M\right], \underbrace{M, M, \ldots, M}_{p-2})=-(M, M) f([N, M], S_{\beta}, \underbrace{M, M, \ldots, M}_{p-2}) .
$$

It turns out that the last equation can be integrated. At first, note that from condition (1.4) it follows that $(M, M)=$ const. Now, one can verify, using Eq.(1.4) and (1.5), the following identity: $H_{, \alpha}=\frac{\partial H}{\partial U^{\alpha}}=(p-1) f([N, M], S_{\alpha}, \underbrace{M, M, \ldots, M}_{p-2})$. Then, we arrive at $Z_{\beta}=-\frac{1}{p-1}(M, M) H_{, \beta}$. Now, one can verify that form $\theta$ is the conservation law of the system under consideration, and the proof is finished.

Example 2.2. Pohlmeier-Lund-Regge system [5],

$$
\begin{gathered}
\Delta^{1}=U_{x y}^{1}+\frac{1}{\sin U^{2}}\left(U_{x}^{1} U_{y}^{2}+U_{y}^{1} U_{x}^{2}\right)=0, \\
\Delta^{2}=U_{x y}^{2}-\frac{\sin U^{2}}{\left(1+\cos U^{2}\right)^{2}} U_{x}^{1} U_{y}^{1}-p \sin U^{2}=0,
\end{gathered}
$$

where $p$ is an arbitrary constant. It will be convenient to write the Lax representation of PLR system in the form (1.3), where

$$
\begin{aligned}
& \widetilde{A}=\left(\begin{array}{ccc}
0 & -\left(\lambda p-\frac{\cos U^{2} U_{x}^{1}}{2 \cos ^{2} \frac{U^{2}}{2}}\right) & -\operatorname{tg} \frac{U^{2}}{2} U_{x}^{1} \\
p \lambda-\frac{\cos U^{2} U_{x}^{1}}{2 \cos ^{2} \frac{U^{2}}{2}} & 0 & U_{x}^{2} \\
\operatorname{tg} \frac{U^{2}}{2} U_{x}^{1} & -U_{x}^{2} & 0
\end{array}\right), \\
& \widetilde{B}=\left(\begin{array}{ccc}
0 & \frac{\cos U^{2}}{\lambda}+\frac{U_{y}^{1}}{2 \cos ^{2} \frac{U^{2}}{2}} & -\frac{\sin U^{2}}{\lambda} \\
-\left(\frac{\cos U^{2}}{\lambda}+\frac{U_{y}^{1}}{2 \cos ^{2} \frac{U^{2}}{2}}\right) & 0 & 0 \\
\frac{\sin U^{2}}{\lambda} & 0 & 0
\end{array}\right) .
\end{aligned}
$$

Choose the following basis $B$ of the Lie algebra $\mathfrak{s o}(3)$

$$
\vec{e}_{1}=\left(\begin{array}{ccc}
0 & 0 & 0 \\
0 & 0 & 1 \\
0 & -1 & 0
\end{array}\right), \vec{e}_{2}=\left(\begin{array}{ccc}
0 & 0 & -1 \\
0 & 0 & 0 \\
1 & 0 & 0
\end{array}\right), \vec{e}_{3}=\left(\begin{array}{ccc}
0 & -1 & 0 \\
1 & 0 & 0 \\
0 & 0 & 0
\end{array}\right) ;
$$

then we have 


$$
\begin{array}{r}
D_{y} \widetilde{A}-D_{x} \widetilde{B}+[\widetilde{A}, \widetilde{B}]=S_{\alpha} \Delta^{\alpha}=\left(\begin{array}{ccc}
0 & \operatorname{tg}^{2} \frac{U^{2}}{2} & -\operatorname{tg} \frac{U^{2}}{2} \\
-\operatorname{tg}^{2} \frac{U^{2}}{2} & 0 & 0 \\
\operatorname{tg} \frac{U^{2}}{2} & 0 & 0
\end{array}\right) \Delta^{1} \\
+\left(\begin{array}{ccc}
0 & 0 & 0 \\
0 & 0 & 1 \\
0 & -1 & 0
\end{array}\right) \Delta^{2} .
\end{array}
$$

Thus, w. r. t. the basis $B$,

$$
\begin{aligned}
& S_{1}=\left(0, \operatorname{tg} \frac{U^{2}}{2},-\operatorname{tg}^{2} \frac{U^{2}}{2}\right), S_{2}=(1,0,0), \\
& M=(0,0, p), N=\left(0, \sin U^{2},-\cos U^{2}\right) .
\end{aligned}
$$

Assume that the 2 -form $f$ is the Killing metric of the Lie algebra $\mathfrak{s o}(3)$ which, w. r. t. the basis $B$, is $\delta_{i j}$ up to a constant factor.

Construct a characteristic of the first order, using expressions (2.10). Assuming that $f$ is the Killing form, we obtain

$$
\begin{gathered}
K=f\left(\left[S_{\alpha}, M\right],\left[S_{\beta}, M\right]\right)=p^{2}\left(\begin{array}{cc}
\tan ^{2} \frac{U^{2}}{2} & 0 \\
0 & 1
\end{array}\right) \\
\widetilde{K}_{\alpha_{\beta}}=f\left(\left[S_{\alpha}, N\right],\left[S_{\beta}, N\right]\right)=\left(\begin{array}{cc}
\tan ^{2} \frac{U^{2}}{2} & 0 \\
0 & 1
\end{array}\right)
\end{gathered}
$$

and

$$
Y_{1}=p^{2} U_{x}^{1} \tan ^{2} \frac{U^{2}}{2}, Y_{2}=p^{2} U_{x}^{2}, \widetilde{Y}_{1}=U_{y}^{1} \tan ^{2} \frac{U^{2}}{2}, \widetilde{Y}_{2}=U_{y}^{2}
$$

One can verify that $Y$ and $\widetilde{Y}$ are the characteristics of the following conservation laws: $\frac{1}{2} p^{2}\left[\left(U_{x}^{1}\right)^{2} \tan ^{2} \frac{U^{2}}{2}+\left(U_{x}^{2}\right)^{2}\right] d x-p^{3} \cos U^{2} d y$ and $\frac{1}{2}\left[\left(U_{x}^{1}\right)^{2} \tan ^{2} \frac{U^{2}}{2}+\left(U_{y}^{2}\right)^{2}\right] d y-p \cos U^{2} d x$, respectively.

Example 2.3. Consider the 3-component system

$$
\begin{gathered}
U_{x y}^{1}+U_{x}^{3} U_{y}^{1} \operatorname{ctg} U^{3}-\frac{1}{\sin U^{3}} U_{y}^{3} U_{x}^{2}+=0, \\
U_{x y}^{2}+U_{y}^{3} U_{x}^{2} \operatorname{ctg} U^{3}-\frac{1}{\sin U^{3}} U_{x}^{3} U_{y}^{1}=0, \\
U_{x y}^{3}+U_{y}^{1} U_{x}^{2} \sin U^{3}-p \sin U^{3}=0,
\end{gathered}
$$

where $p$ is an arbitrary constant. This system admits the Lax representation of the form (1.3), where [3], [2]: 


$$
\begin{aligned}
& \widetilde{A}=\left(\begin{array}{ccc}
0 & i \lambda M^{3} & -i \lambda M^{2} \\
-\lambda M^{3} & 0 & i \lambda M^{1} \\
i \lambda M^{2} & -i \lambda M^{1} & 0
\end{array}\right) \\
& \widetilde{B}=\left(\begin{array}{ccc}
0 & \frac{i}{\lambda}-\left(\cos U^{3} U_{y}^{1}+U_{y}^{2}\right) & -b_{31} \\
-\frac{i}{\lambda}+\left(\cos U^{3} U_{y}^{1}+U_{y}^{2}\right) & 0 & b_{23} \\
b_{31} & -b_{23} & 0
\end{array}\right) \\
& M^{1}=p \sin U^{3} \sin U^{2}, M^{2}=-p \sin U^{3} \cos U^{2}, M^{3}=p \cos U^{3}, \\
& b_{31}=\sin U^{3} \cos U^{2} U_{y}^{1}-\sin U^{2} U_{y}^{3}, b_{23}=-\cos U^{2} U_{y}^{3}-\sin U^{2} \sin U^{3} U_{y}^{1} .
\end{aligned}
$$

Consider the same basis $B$ and the same 2-form $\mathrm{f}$ as in example 1. Then, we find $S_{1}=$ $\left(\sin U^{2} \sin U^{3},-\cos U^{2} \sin U^{3}, \cos U^{3}\right), S_{2}=(0,0,1), S_{3}=\left(\cos U^{2}, \sin U^{2}, 0\right)$.

Again, using (2.10) and assuming $f$ to be the Killing form, one can find

$$
\begin{gathered}
K_{\alpha \beta}=f\left(\left[S_{\alpha}, M\right],\left[S_{\beta}, M\right]\right)=p^{2}\left(\begin{array}{ccc}
0 & 0 & 0 \\
0 & \sin ^{2} U^{3} & 0 \\
0 & 0 & 1
\end{array}\right), \\
\widetilde{K}_{\alpha \beta}=f\left(\left[S_{\alpha}, N\right],\left[S_{\beta}, N\right]\right)=-\left(\begin{array}{ccc}
\sin ^{2} U^{3} & 0 & 0 \\
0 & 0 & 0 \\
0 & 0 & 1
\end{array}\right) .
\end{gathered}
$$

Thus, according to the Theorem 2, the set $Y_{\alpha}=p^{2}\left(0, \sin ^{2} U^{3} U_{x}^{2}, U_{x}^{3}\right)$ is a characteristic of the conservation law. Indeed, the corresponding conservation law is

$$
\theta=-p^{3} \cos U^{3} d y+\frac{p^{2}}{2} d x\left[\left(U_{x}^{3}\right)^{2}+\left(U_{x}^{2} \sin U^{3}\right)^{2}\right] .
$$

Analogously, one can see that $\widetilde{Y}_{\alpha}=-\left(\sin ^{2} U^{3} U_{y}^{1}, 0, U_{y}^{3}\right)$ and corresponding conservation law is of the form

$$
\widetilde{\theta}=p \cos U^{3} d x-\frac{d y}{2}\left[\left(U_{y}^{3}\right)^{2}+\left(U_{y}^{1} \sin U^{3}\right)^{2}\right]
$$

\section{Conclusions}

In this paper, the geometric meaning of some tensor fields constructed by the Lax representation of chiral-type systems is shown. The formula for characteristics of the first order of the conservation laws for the chiral-type systems admitting $\mathfrak{g}$-valued Lax representation $(\operatorname{dim} \mathfrak{g}=3)$ has been proved. It seems interesting to find similar formulas for characteristics of higher orders. Such fields may turn out to be useful to obtain a criterion of existence of a Lax pair. 


\section{Acknowledgments}

The author is grateful to E.V. Ferapontov, M.I. Kuznetsov, Y.V. Tuzov, and A.V. Zhidkov for useful discussions. The author is thankful to the referees for useful remarks. This work was supported by the project 1410 of Russian Ministry of Education and Science.

\section{References}

[1] A.V. Balandin, Conservation Laws of Chiral-Type Systems, Vestnik Nizhegorodskogo Universiteta 6(1) (2013) 153-156 (in Russian).

[2] A.V. Balandin, Characteristics of Conservation Laws of Chiral-Type Systems, Lett. Math. Phys. 105 (2015) 27-43 (arXiv:1310.5218).

[3] A.V. Balandin, O.N. Pakhareva and G.V. Potemin, Lax representation of the chiral-type field equations, Phys. Lett. A. 23 (2001) 168-176.

[4] D.K. Demskoi, V.G. Marikhin and A.G. Meshkov, Lax representations for triplets of two-dimensional scalar fields of the chiral type, Theoretical and Mathematical Physics 148 (2006) 1034-1048.

[5] F. Lund and T. Regge, Unified approach to strings and vortices with soliton solutions, Phys.Rew. D. 14 (1976) 1524-1535.

[6] M. Marvan, On zero-curvature representations of partial differential equations, 5th International Conference on Differential Geometry and Its Applications, (Opava, Czech Republic, 1993) 103-122, (http://www.emis.de/proceedings/5ICDGA).

[7] P. Olver, Applications of Lie Groups to Differential Equations, (2nd ed., Springer, New York, 1993)

\section{Appendix}

Here, we prove theorem 1.

According to Eq. (1.4),(2.1) rewrite $\nabla_{\gamma} K_{\alpha_{1} \alpha_{2} \ldots \alpha_{k}}$ by the following way

$$
\begin{aligned}
& \nabla_{\gamma} K_{\alpha_{1} \alpha_{2} \ldots \alpha_{k}}=f(\left[\nabla_{\gamma} S_{\alpha_{1}}, M\right],\left[S_{\alpha_{2}}, M\right], \ldots,\left[S_{\alpha_{k}}, M\right], \underbrace{M, \ldots, M}_{p-k}) \\
&+f(\left[S_{\alpha_{1}}, M, \gamma\right],\left[\nabla_{\gamma} S_{\alpha_{2}}, M\right], \ldots,\left[S_{\alpha_{k}}, M\right], \underbrace{M, \ldots, M}_{p-k})+\ldots \\
&+f(\left[S_{\alpha_{1}}, M\right],\left[S_{\alpha_{2}}, M\right], \ldots,\left[S_{\alpha_{k}}, M, \gamma\right], \underbrace{M, \ldots, M}_{p-k}) \\
&+(p-k) f(\left[S_{\alpha_{1}}, M\right],\left[S_{\alpha_{2}}, M\right], \ldots,\left[S_{\alpha_{k}}, M\right], M, \gamma, \underbrace{M, M, \ldots, M}_{p-k-1}) \\
&= f(\left[\left[B_{\gamma}, S_{\alpha_{1}}\right], M\right],\left[S_{\alpha_{2}}, M\right], \ldots,\left[S_{\alpha_{k}}, M\right], \underbrace{M, \ldots, M}_{p-k}) \\
& \quad-f(\left[D_{\alpha_{1} \gamma}, M\right],\left[S_{\alpha_{2}}, M\right], \ldots,\left[S_{\alpha_{k}}, M\right], \underbrace{M, \ldots, M}_{p-k}) \\
&+ f(\left[S_{\alpha_{1}},\left[B_{\gamma}, M\right]\right],\left[S_{\alpha_{2}}, M\right], \ldots,\left[S_{\alpha_{k}}, M\right], \underbrace{M, \ldots, M}_{p-k}) \\
&+
\end{aligned}
$$


A.V. Balandin / Tensor fields defined by Lax representations

$$
\begin{aligned}
+ & \begin{array}{l}
f(\left[S_{\alpha_{1}}, M\right],\left[\left[B_{\gamma}, S_{\alpha_{2}}\right], M\right], \ldots,\left[S_{\alpha_{k}}, M\right], \underbrace{M, \ldots, M}_{p-k}) \\
+f(\left[S_{\alpha_{1}}, M\right],\left[D_{\alpha_{2} \gamma}, M\right], \ldots,\left[S_{\alpha_{k}}, M\right], \underbrace{M, \ldots, M}_{p-k}) \\
+f(\left[S_{\alpha_{1}}, M\right],\left[S_{\alpha_{2}},\left[B_{\gamma}, M\right]\right], \ldots,\left[S_{\alpha_{k}}, M\right], \underbrace{M, \ldots, M}_{p-k}) \\
+\ldots+(p-k) f(\left[S_{\alpha_{1}}, M\right],\left[S_{\alpha_{2}}, M\right], \ldots,\left[S_{\alpha_{k}}, M\right],\left[B_{, \gamma}, M\right] \underbrace{M, \ldots, M}_{p-k-1})
\end{array}
\end{aligned}
$$

Now, one can see that the underlined terms vanish due to Ad-invariancy of the form $f$. Next, one can obtain from Eq. (1.4) the following identity

$$
\left[2 B_{[\alpha, \gamma]}, M\right]=-\left[\left[B_{\alpha}, B_{\gamma}\right], M\right] .
$$

Taking into account Eq. (2.2), one can find that $\left[D_{\alpha \beta}, M\right]=0$. The proof is complete. 